\title{
DRG-Krankenhäuser im Vergleich
}

\author{
Elektronisches Zusatzmaterial Die elektronische Version dieses Kapitels enthält Zusatzmaterial, das den Nutzern zur \\ Verfügung steht unter https://doi.org/10.1007/978-3-662-58225-1_20 und unter www.springer.com/book/9783662582244. \\ Jörg Friedrich \\ (c) Der/die Autor(en) 2019 \\ J. Klauber et al. (Hrsg.), Krankenhaus-Report 2019 \\ https://doi.org/10.1007/978-3-662-58225-1_20
}

\section{Zusammenfassung}

Das Directory deutscher Krankenhäuser bietet eine jährlich aktualisierte Übersicht stationärer Leistungserbringer. Die Darstellung umfasst unter anderem Informationen zur Struktur des vereinbarten Leistungsangebots, zum Grad der Spezialisierung, zur regionalen Marktpositionierung und Wettbewerbssituation sowie Informationen zur Ergebnisqualität nach dem Verfahren Qualitätssicherung mit Routinedaten (QSR). Insgesamt finden mehr als 1.400 Krankenhäuser Eingang, zu denen eine Budgetvereinbarung für das Jahr 2017 oder QSR-Behandlungsergebnisse vorliegen.

The Directory of German Hospitals provides an annually updated overview of inpatient service providers. It includes information on the structure of the agreed range of services, the degree of specialisation, regional market positioning and competitive situation as well as information on the outcome quality according to QSR, a method of quality reporting based on routine data of health care funds. In total, more than 1,400 hospitals have been included for which a budget agreement for the year 2017 or QSR treatment outcomes are available.

\subsection{Krankenhaus-Directory}

Das jährliche Directory deutscher Krankenhäuser stellt Eckdaten aus den Aufstellungen der Entgelte und Budgetermittlung (AEB) gemäß Krankenhausentgeltgesetz (KHEntgG) dar. Den Darstellungen liegen Vereinbarungsdaten und nicht die tatsächlich erbrachten Leistungen der jeweiligen Einrichtung zugrunde. Insgesamt finden mehr als 1.400 Krankenhäuser Eingang, zu denen eine Vereinbarung oder QSR-Behandlungsergebnisse vorliegen. Das Krankenhaus-Directory finden Sie unter www. springer.com/book/9783662582244.

Die einzelnen Spalten des Directories haben folgende Bedeutung:

\section{- Krankenhausname}

Mit einem * gekennzeichnete Einrichtungen haben nach Abschluss der Vereinbarung 2017 mit einem anderen Krankenhaus fusioniert oder wurden geschlossen.

\section{- Betten}

Jedes Krankenhaus wird nach seiner Bettenzahl klassifiziert und einer von sechs Kategorien zugeordnet. Die verwendeten Symbole bedeuten Folgendes:

$$
\begin{aligned}
& <50=\text { unter } 50 \text { Betten } \\
& <100=50 \text { bis unter } 100 \text { Betten } \\
& <200=100 \text { bis unter } 200 \text { Betten } \\
& <500=200 \text { bis unter } 500 \text { Betten } \\
& <1.000=500 \text { bis unter } 1.000 \text { Betten } \\
& >1.000=\text { über } 1.000 \text { Betten }
\end{aligned}
$$

Die Angaben stammen überwiegend aus dem Jahr 2017, andernfalls aus den Vorjahren.

\section{- Träger}

In dieser Spalte wird die Trägerschaft des Krankenhauses mit folgenden Abkürzungen geschlüsselt:

ö für öffentlich

fg für freigemeinnützig

$\mathrm{p}$ für privat 


\section{- Z-Bax (Zahlbasisfallwert)}

Der Basisfallwert ist der Eurobetrag, der multipliziert mit der Bewertungsrelation den Preis einer DRG-Fallpauschale festlegt. Für die Vergütung der Krankenhausfälle einer laufenden Periode ist der Zahlbasisfallwert maßgeblich, der auch Transferzahlungen aus vergangenen Perioden, sogenannte Erlösausgleiche, berücksichtigt. Außerdem dient der Zahlbasisfallwert auch der sachgerechten Umsetzung unterjährig vereinbarter Gesamtjahreswerte. Der gemittelte Zahlbasisfallwert (Z-Bax) ist ein Indikator für das tatsächlich herrschende Preisniveau des Jahres für Krankenhausleistungen, die nach DRGs vergütet werden. ${ }^{1}$ Der Z-Bax umfasst alle relevanten $\mathrm{Zu}$ - und Abschlagstatbestände. Deren Vergütung wird ebenfalls je Bewertungsrelation, also analog dem Basisfallwert ausgedruckt (Friedrich et al. 2010). ${ }^{2}$

In der Spalte für den Basisfallwert ist ein „BE“ $\mathrm{zu}$ finden, wenn das gesamte Krankenhaus 2017 keine DRG-Entgelte vereinbart hat, z. B. auf Basis der Vereinbarung zur Bestimmung von Besonderen Einrichtungen 2017, und es somit als Ganzes von der Anwendung der DRG-Fallpauschalen ausgenommen ist.

\section{- Casemix}

Der Casemix ist die Summe aller Bewertungsrelationen einer Einrichtung. Jedes Krankenhaus wird

1 Der bundesweite Z-Bax steht wochenaktuell unter www. wido.de als Download zur Verfügung.

2 Alle fallbezogenen Zuschläge werden bei Anrechnung im Z-Bax durch den vereinbarten CMI des Hauses dividiert. Der tagesbezogene Investitionszuschlag wird näherungsweise über die mittlere Verweildauer der vereinbarten DRGs ermittelt. Die berücksichtigten Zuschläge im Z-Bax lauten z. Zt.: Zuschlag Abschaffung des Arztes im Praktikum (AiP), Zuschlag Finanzierung von Arbeitszeitverbesserungen (AZV), Zuschlag Ausbildungsfinanzierung, Investitionszuschlag, Zuschlag Qualitätssicherungszuschlag, Sicherstellungszuschlag, Zuschlag Zentren und Schwerpunkte, Zuschlag Vorhaltekosten Besonderer Einrichtungen, Abschlag Tariferhöhung, Abschlag für Anschubfinanzierung Integrierter Versorgung, Abschlag für vereinbarte Mehrleistungen, Fixkostendegressionsabschlag, Abschlag Nichtteilnahme am Datenträgeraustausch, Abschlag Nichtteilnahme an Notfallversorgung, Sanierungsabschlag, Ausgleiche, Kappung, Versorgungszuschlag, Konvergenzverlängerung, Zuschlag für Mehrkosten aus GBA-Richtlinien und Konvergenz Besondere Einrichtungen. anhand des vereinbarten Casemix klassifiziert und einer von sechs Kategorien zugeordnet. Die verwendeten Symbole bedeuten Folgendes:

$$
\begin{aligned}
<1.000= & \text { unter } 1.000 \text { Bewertungsrelationen } \\
<5.000= & 1.000 \text { bis unter 5.000 Bewertungs- } \\
& \text { relationen } \\
<10.000= & 5.000 \text { bis unter 10.000 Bewertungs- } \\
& \text { relationen } \\
<20.000= & 10.000 \text { bis unter } 20.000 \text { Bewertungs- } \\
& \text { relationen } \\
<50.000= & 20.000 \text { bis unter } 50.000 \text { Bewertungs- } \\
& \text { relationen } \\
>50.000= & \text { über } 50.000 \text { Bewertungsrelationen }
\end{aligned}
$$

\section{- CMI (Casemix-Index)}

Der Casemix-Index (CMI) beschreibt die mittlere Fallschwere eines Krankenhauses. Er berechnet sich aus dem Quotienten des Casemix (Summe aller Bewertungsrelationen eines Krankenhauses) und der Gesamtzahl der über DRGs abgerechneten Fälle eines Krankenhauses. Der hier ausgewiesene CMI enthält keine teilstationären DRGs.

\section{- Abw. CMI Land}

Für jede Einrichtung wird der individuelle CMI mit dem entsprechenden Landeswert verglichen. Die Abweichungen sind mit folgenden Symbolen gekennzeichnet:

$+++=$ Abweichung vom Landeswert von über $20 \%$

$++\quad=$ Abweichung vom Landeswert von $10 \%$ bis unter $20 \%$

$+\quad=$ Abweichung vom Landeswert von $0 \%$ bis unter $10 \%$

= Abweichung vom Landeswert von $0 \%$ bis über $-10 \%$

- - $\quad=$ Abweichung vom Landeswert von $-10 \%$ bis über $-20 \%$

$---=$ Abweichung vom Landeswert von unter $-20 \%$

\section{- Vereinbarter Spezialisierungsgrad im DRG-Bereich (Gini-Koeffizient)}

Die Werte beschreiben den Grad der Spezialisierung für DRG-Leistungen des jeweiligen Krankenhauses anhand des Gini-Koeffizienten. Die Ermittlung erfolgt auf der Ebene Basis-DRG (A-DRG). Der Gini-Koeffizient ist eine Maßzahl für die (Un) 
gleichverteilung innerhalb einer Grundgesamtheit. Sind die Leistungen eines Krankenhauses über alle Basis-DRGs gleich verteilt, liegt keine Spezialisierung vor. Verteilen sich die Fälle auf nur wenige Basis-DRGs und ist die Verteilung somit sehr ungleich, so kann das Krankenhaus als spezialisiert gelten. Ein Gini-Koeffizient von 1 resultierte aus einer maximalen Spezialisierung auf nur eine Leistung, ein Wert von 0 entspräche einer identischen Fallzahl in allen Basis-DRGs.

Aus dem Grad der Spezialisierung der Krankenhäuser lassen sich nur wenige Rückschlüsse auf die Zentralisierung der Leistungserbringung ziehen. - Tab. 20.1 und - Tab. 20.2 illustrieren die Verteilung der Fallzahlen je vollstationäre Basis-DRG (s. u.) der operativen bzw. der medizinischen Partition auf die vorliegenden Vereinbarungen. Die Darstellung erfolgt nach Fallzahlquintilen. Die Spalten zum ersten Quintil geben z. B. darüber Auskunft, welchen Anteil die $20 \%$ der Krankenhäuser mit den größten Fallzahlen am Gesamtaufkommen haben. Die Spalten zum fünften Quintil geben u. a. Hinweise, in welchen Basis-DRGs die 20 \% der Krankenhäuser mit den geringsten Fallzahlen die entsprechende Leistung nur sehr selten erbringen. Die Darstellung beschränkt sich auf die jeweils 25 fallzahlstärksten Basis-DRGs.

\section{- Leistungsdichte Basis-DRGs}

Es wird jeweils angegeben, mit wie vielen BasisDRGs (A-DRGs) jeweils $25 \%$, $50 \%$ und $75 \%$ aller Leistungen eines Hauses erreicht werden. BasisDRGs stellen eine Obergruppe für eine oder mehrere DRGs dar, die durch die gleichen Diagnosenund/oder Prozedurencodes definiert sind. DRGs innerhalb einer Basis-DRG unterscheiden sich in ihrem Ressourcenverbrauch bzw. ihres Schweregrads. In der G-DRG Version 2017 gibt es 558 BasisDRGs, davon zwei nicht bewertete Fehler-DRGs und eine teilstationäre DRG.

\section{- TOP 3 MDC}

In einer weiteren Annäherung an das DRG-Leistungsspektrum eines Hauses werden die fünf jeweils stärksten Hauptdiagnosegruppen (MDCs; Major Diagnostic Category) mit ihrer Nummer sowie dem jeweiligen Prozentanteil an sämtlichen
DRG-Leistungen dokumentiert. Die Nummern der MDCs bedeuten Folgendes:

-1 Pre-MDC

1. Krankheiten und Störungen des Nervensystems

2. Krankheiten und Störungen des Auges

3. Krankheiten und Störungen im HNO-Bereich

4. Krankheiten und Störungen der Atmungsorgane

5. Krankheiten und Störungen des Kreislaufsystems

6. Krankheiten und Störungen der Verdauungsorgane

7. Krankheiten und Störungen am hepatobiliären System und Pankreas

8. Krankheiten und Störungen am MuskelSkelett-System und Bindegewebe

9. Krankheiten und Störungen an Haut, Unterhaut und Mamma

10. Endokrine, Ernährungs- und Stoffwechselkrankheiten

11. Krankheiten und Störungen der Harnorgane

12. Krankheiten und Störungen der männlichen Geschlechtsorgane

13. Krankheiten und Störungen der weiblichen Geschlechtsorgane

14. Schwangerschaft, Geburt und Wochenbett

15. Neugeborene

16. Krankheiten des Blutes, der blutbildenden Organe und des Immunsystems

17. Hämatologische und solide Neubildungen

18. Infektiöse und parasitäre Krankheiten

19. Psychiatrische Krankheiten und Störungen

20. Alkohol- und Drogengebrauch und alkoholund drogeninduzierte psychische Störungen

21. Verletzungen, Vergiftungen und toxische Nebenwirkungen von Drogen und Medikamenten

22. Verbrennungen

23. Faktoren, die den Gesundheitszustand beeinflussen und andere Inanspruchnahmen des Gesundheitswesens

24. Sonstige DRGs 


\begin{tabular}{|c|c|c|c|c|c|c|c|c|c|c|c|}
\hline & 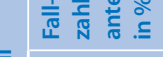 & n & $\sigma$ & 0 & - & 0 & $\sigma$ & $\sigma$ & 0 & o & 。 \\
\hline & 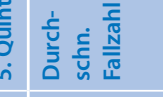 & $\stackrel{ }{\cong}$ & $\hat{m}$ & $n$ & $\infty$ & m & $\tilde{m}$ & m & f & m & m \\
\hline & 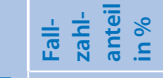 & $=$ & & $\stackrel{m}{-}$ & ^ & $\sigma$ & 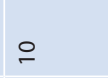 & $a$ & $\stackrel{m}{\longrightarrow}$ & $\sigma$ & N \\
\hline & 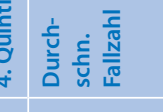 & $\stackrel{\infty}{\varrho}$ & ส & $\stackrel{m}{=}$ & 함 & $\hat{m}$ & r & $\stackrel{\infty}{\wedge}$ & $\stackrel{ \pm}{\stackrel{ \pm}{+}}$ & $\stackrel{\infty}{m}$ & $\bar{N}$ \\
\hline & 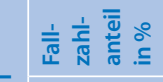 & $\stackrel{n}{\sim}$ & 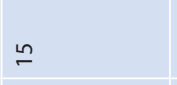 & $\stackrel{\infty}{-}$ & $\stackrel{9}{-1}$ & $\simeq$ & $\cong$ & \pm & $\stackrel{\infty}{\simeq}$ & $\Xi$ & $\wedge$ \\
\hline & 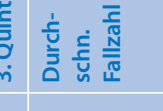 & సิ & $\underset{\mathcal{I}}{\check{1}}$ & ติ & 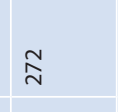 & $\stackrel{\circ}{\rightleftharpoons}$ & $\stackrel{\stackrel{ \pm}{\simeq}}{ }$ & $\stackrel{\tilde{N}}{\simeq}$ & $\underset{g}{\mathbb{g}}$ & $\bar{m}$ & $\stackrel{\infty}{\infty}$ \\
\hline & 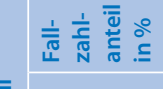 & ম & $\approx$ & đ & $\stackrel{\infty}{\sim}$ & $\stackrel{\sim}{\sim}$ & $\stackrel{d}{\Lambda}$ & $\tilde{\sim}$ & $\stackrel{ \pm}{N}$ & 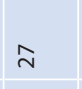 & $\bar{N}$ \\
\hline & 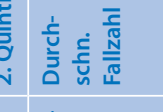 & $\stackrel{\infty}{m}$ & ஓ् & $\stackrel{\sim}{\sim}$ & $\stackrel{N}{\sigma}$ & సి & $\stackrel{\bar{\infty}}{-}$ & $\hat{a}$ & б̄ & ్ָ & $\stackrel{\infty}{\stackrel{\infty}{\circ}}$ \\
\hline & $=$ & 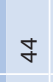 & in & q & f & in & ร & g & $\stackrel{\infty}{m}$ & in & R \\
\hline & 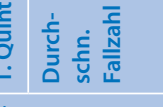 & $\hat{m}$ & $\bar{g}$ & ্̊ & $\widehat{\stackrel{\iota}{0}}$ & $\bar{n}$ & 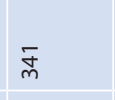 & $\frac{\infty}{7}$ & 商 & $\bar{n}$ & ठे \\
\hline & 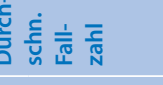 & 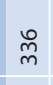 & $\bar{\sigma}$ & $\stackrel{\infty}{-}$ & ๙ิ & $\stackrel{\curvearrowright}{\check{1}}$ & $\underline{5}$ & $\stackrel{?}{\stackrel{2}{2}}$ & $\stackrel{\text { 足 }}{\stackrel{2}{2}}$ & مิ & $\tilde{\alpha}$ \\
\hline & 密 & f & $N$ & $N$ & q & ț & $\kappa$ & 8 & 8 & in & f \\
\hline & 颉 & in & $\underset{\infty}{\infty}$ & $\begin{array}{c}\bar{\infty} \\
\infty\end{array}$ & ஓ̊ & $\stackrel{\infty}{\wedge}$ & $\bar{\sigma}$ & $\frac{\sigma}{\infty}$ & $\stackrel{\infty}{\infty}$ & ̊̊ & 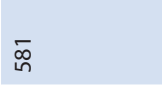 \\
\hline & 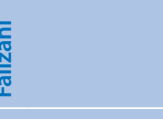 & 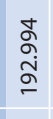 & $\begin{array}{l}\stackrel{\circ}{\circ} \\
\text { oे } \\
\stackrel{0}{0}\end{array}$ & $\begin{array}{l}\tilde{N} \\
\substack{\infty \\
\infty \\
\sim \\
\sim}\end{array}$ & 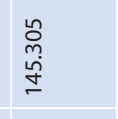 & 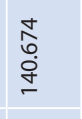 & $\begin{array}{l}\hat{o} \\
\text { ò } \\
\text { g }\end{array}$ & 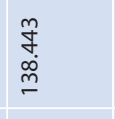 & $\begin{array}{l}\stackrel{m}{m} \\
\stackrel{\dot{p}}{m} \\
\stackrel{m}{m}\end{array}$ & $\begin{array}{l}\stackrel{0}{0} \\
\stackrel{m}{m} \\
\stackrel{m}{m}\end{array}$ & 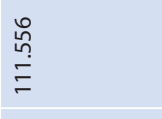 \\
\hline & 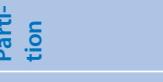 & 0 & $\circ$ & $\circ$ & $\circ$ & $\circ$ & 0 & 0 & 0 & 0 & 0 \\
\hline & 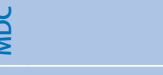 & \pm & $\infty$ & 0 & $=$ & $\infty$ & $\infty$ & $\infty$ & $\wedge$ & in & m \\
\hline & 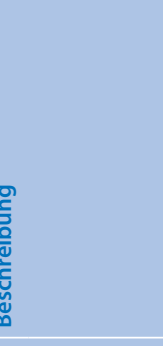 & & 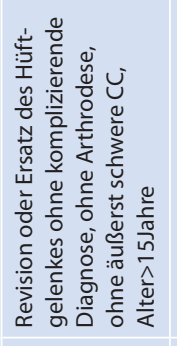 & 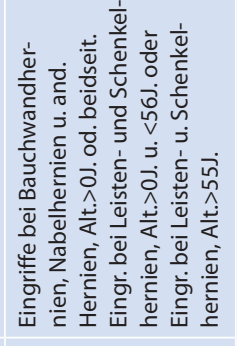 & 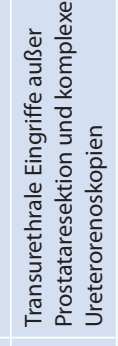 & 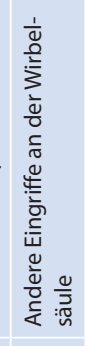 & 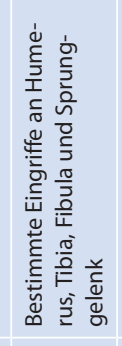 & 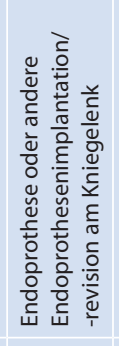 & 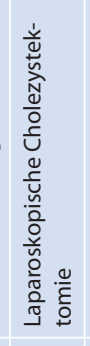 & 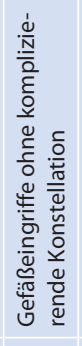 & 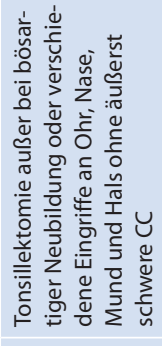 \\
\hline & & $\overline{8}$ & 王 & đ্ড & ฐิ & $\stackrel{ }{\varrho}$ & $\stackrel{m}{=}$ & \pm & $\stackrel{\infty}{\text { 오 }}$ & ڤે & סิ \\
\hline
\end{tabular}


ఫ்

$\stackrel{n}{\sim} \stackrel{m}{\sim} \cong$

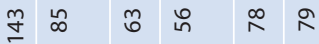

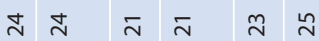

สิ ิํำ

g f in 品 禾

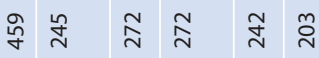

$\stackrel{\infty}{\circ}$ 의

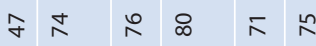

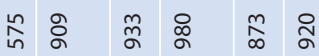

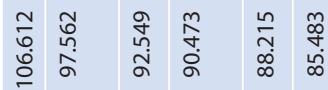

0000000

$\begin{array}{llllll}\text { in } & \infty & \infty & \sigma & 0 & \infty\end{array}$
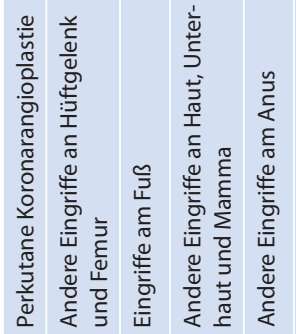

帘

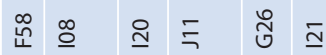

g)

$\stackrel{\infty}{\infty}$

$\stackrel{\infty}{\check{\infty}}$

$\stackrel{ }{\sim}$

$\stackrel{n}{=}$

$\stackrel{\infty}{\infty}$

$\stackrel{2}{2}$

ก

ఫ

กิ เั

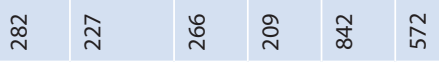

y

$\stackrel{\infty}{+}$

ळ

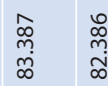

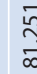

กิ

$\stackrel{\infty}{\curvearrowright}$

$\underset{\substack{0 \\ \stackrel{0}{\circ}}}{\stackrel{n}{\wedge}}$

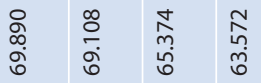

○

○

这

竞 


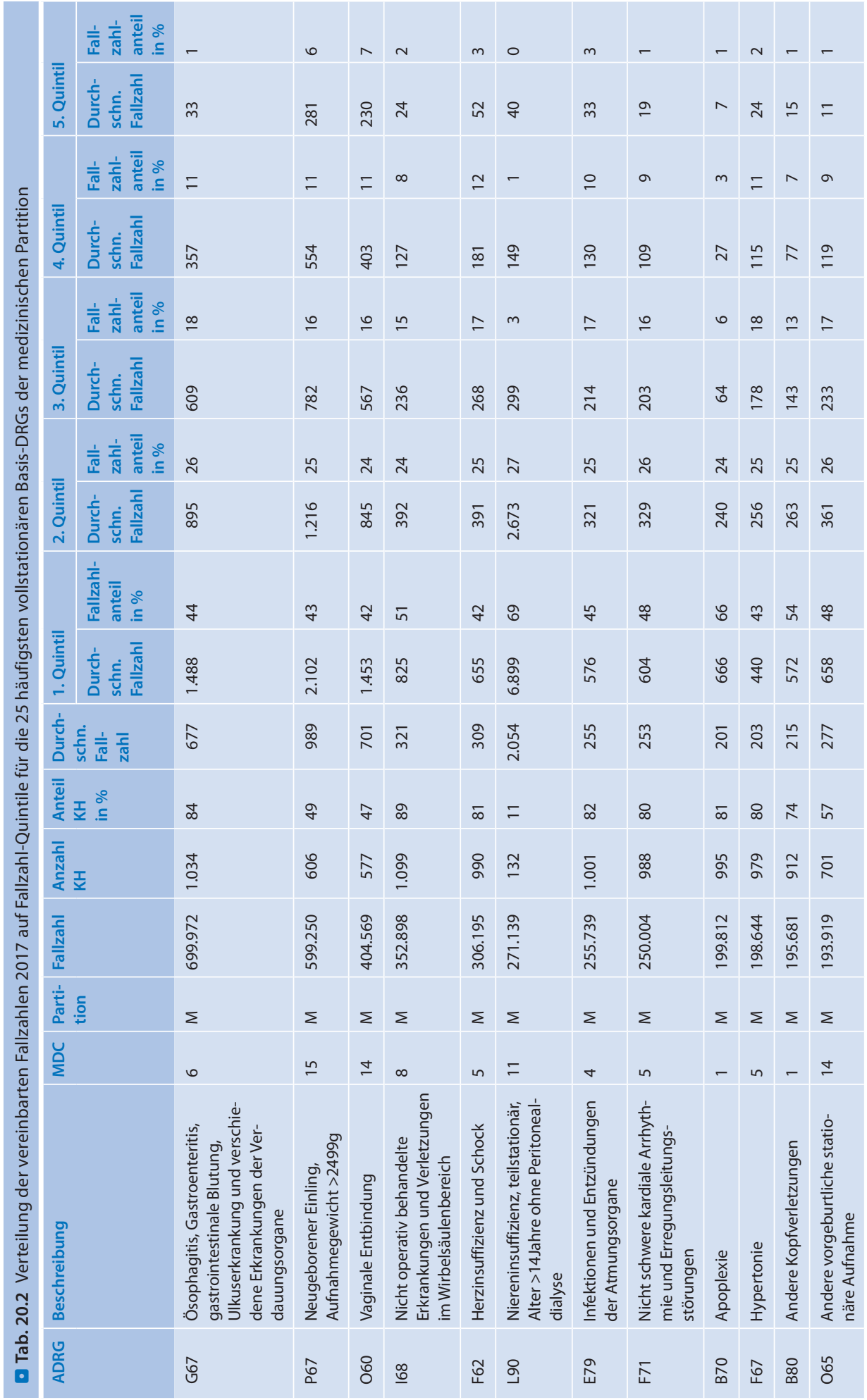




\begin{tabular}{|c|c|c|c|c|c|c|c|c|c|c|c|c|c|}
\hline$m$ & $\sim$ & - & $\sim$ & $\sim$ & $N$ & - & - & $N$ & - & $\sim$ & $N$ & - & \\
\hline$\stackrel{\sim}{\sim}$ & $\stackrel{\sigma}{\longrightarrow}$ & $a$ & $\stackrel{\infty}{\llcorner}$ & $\stackrel{\circ}{\circ}$ & $\stackrel{0}{\circ}$ & 0 & $\stackrel{ }{-}$ & $=$ & $m$ & $\simeq$ & 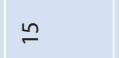 & $\infty$ & \\
\hline 음 & $a$ & $\sigma$ & 으 & $=$ & $a$ & $m$ & $\infty$ & $\wedge$ & $N$ & $\infty$ & $a$ & $a$ & \\
\hline n & $\curvearrowright$ & $\stackrel{m}{m}$ & ঞ & ○ & $\mathscr{\wp}$ & $\ddot{\sim}$ & $\stackrel{\infty}{\llcorner}$ & भ & $\stackrel{m}{\longrightarrow}$ & in & 8 & กี & \\
\hline$\stackrel{\bullet}{\circ}$ & $\stackrel{n}{\sim}$ & $a$ & $\therefore$ & $\stackrel{\infty}{\sim}$ & $\stackrel{\circ}{ }$ & $\wedge$ & $\stackrel{n}{\sim}$ & $\stackrel{m}{\sim}$ & $\wedge$ & \pm & $\stackrel{\circ}{\circ}$ & 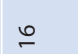 & \\
\hline$\stackrel{\text { ำ }}{n}$ & $\stackrel{\stackrel{n}{m}}{\sim}$ & $\curvearrowright$ & $\tilde{m}$ & $\stackrel{m}{\sim}$ & $\underset{\Xi}{\simeq}$ & g & $\underset{O}{ \pm}$ & $\bar{\infty}$ & $f$ & $\widetilde{\infty}$ & อิ & $\bar{\sigma}$ & \\
\hline$\stackrel{\Delta}{\sim}$ & $\stackrel{\bullet}{\sim}$ & $\stackrel{\sim}{\sim}$ & $\stackrel{\sim}{\sim}$ & $\stackrel{\sim}{N}$ & $\stackrel{\sim}{N}$ & $\stackrel{\circ}{\circ}$ & $\stackrel{\sim}{\sim}$ & $\stackrel{\sim}{\sim}$ & $\stackrel{\sim}{\sim}$ & $\approx$ & $\stackrel{\sim}{\sim}$ & $\stackrel{\mathscr{N}}{\sim}$ & \\
\hline$\underset{\approx}{\approx}$ & $\stackrel{\infty}{\sim}$ & $\frac{a}{\sim}$ & 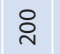 & $\stackrel{\infty}{\infty}$ & ঃ্ & $\stackrel{ }{\beth}$ & $\stackrel{\stackrel{n}{上}}{=}$ & $\underline{n}$ & $\stackrel{\circ}{\llcorner}$ & $\tilde{m}$ & $\stackrel{n}{6}$ & $\stackrel{\infty}{m}$ & \\
\hline f & $\stackrel{\infty}{q}$ & 8 & \& & $\tilde{q}$ & f & $\stackrel{\aleph}{\wedge}$ & in & in & 乞 & 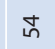 & fo & $\stackrel{\infty}{\sigma}$ & \\
\hline 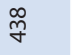 & $\underset{\forall}{\widetilde{\gamma}}$ & in & $\underset{m}{\mathbb{J}}$ & $\frac{\circ}{m}$ & gे & $\underset{H}{\mathbb{H}}$ & $\hat{\mathrm{O}}$ & 鬲 & $\hat{q}$ & $\stackrel{\infty}{m}$ & $\stackrel{\infty}{\stackrel{\infty}{~}}$ & $\bar{\lesssim}$ & \\
\hline$\stackrel{\infty}{\infty}$ & $\stackrel{\swarrow}{\curvearrowleft}$ & $\stackrel{N}{\Sigma}$ & $\stackrel{n}{n}$ & $\stackrel{\mathscr{f}}{\underline{T}}$ & $\stackrel{n}{n}$ & $\underset{g}{g}$ & $\stackrel{m}{\underset{q}{q}}$ & $\stackrel{\searrow}{\sim}$ & 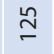 & $\bar{ָ}$ & 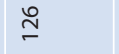 & $\stackrel{\cong}{\cong}$ & \\
\hline$\triangleright$ & $\bar{\infty}$ & $\stackrel{\circ}{\wedge}$ & $\infty$ & $\varpi_{\infty}$ & $\infty$ & $\stackrel{\infty}{\wedge}$ & $\stackrel{\infty}{\wedge}$ & $\bar{\infty}$ & $\stackrel{\curvearrowright}{\wedge}$ & $\bar{\infty}$ & $\stackrel{2}{\wedge}$ & $\infty$ & \\
\hline సે & $\underset{\sim}{\stackrel{\circ}{0}}$ & $\stackrel{m}{n}$ & $\stackrel{\text { ڤno }}{\circ}$ & $\stackrel{\sigma}{\circ}$ & $\bar{\infty}$ & กू & চ̊ & สু & ด̊ & ณू & ñ & 员 & \\
\hline 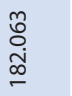 & $\begin{array}{l}\frac{n}{\infty} \\
\stackrel{\infty}{上}\end{array}$ & $\begin{array}{l}\infty \\
\infty \\
\stackrel{0}{0} \\
\stackrel{0}{0}\end{array}$ & $\begin{array}{l}\stackrel{0}{1} \\
\text { ஸn } \\
\stackrel{n}{n}\end{array}$ & $\begin{array}{l}\hat{\infty} \\
\prod_{0} \\
\stackrel{d}{+}\end{array}$ & 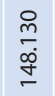 & 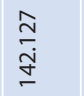 & $\begin{array}{l}\text { ర్ } \\
\text { ôे } \\
\text { m. }\end{array}$ & 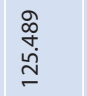 & 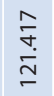 & 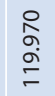 & $\begin{array}{l}\hat{o} \\
\stackrel{0}{0} \\
\stackrel{\Xi}{=}\end{array}$ & 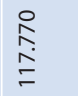 & \\
\hline$\Sigma$ & $\Sigma$ & $\Sigma$ & $\Sigma$ & $\Sigma$ & $\Sigma$ & $\Sigma$ & $\Sigma$ & $\Sigma$ & $\Sigma$ & $\Sigma$ & $\Sigma$ & $\Sigma$ & \\
\hline$\sigma$ & ナ & $=$ & in & $\stackrel{\circ}{\circ}$ & $=$ & $\sigma$ & $a$ & $m$ & - & 으 & 0 & $a$ & \\
\hline 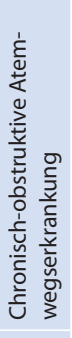 & 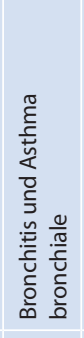 & 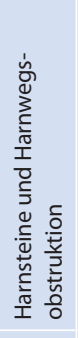 & 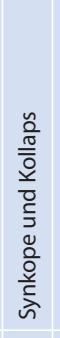 & 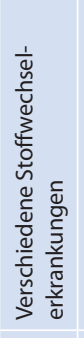 & 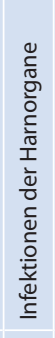 & 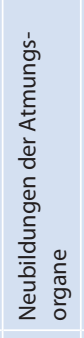 & 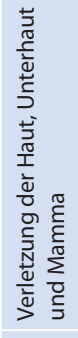 & 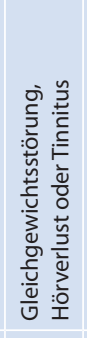 & 产 & 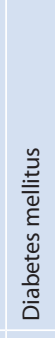 & 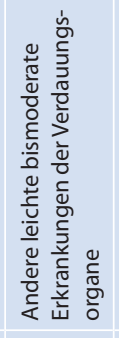 & 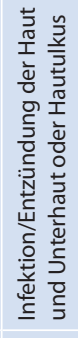 & 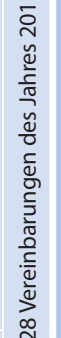 \\
\hline 怘 & 임 & む্ & $\underset{\mathbf{L}}{m}$ & $\underline{\tilde{\sigma}}$ & $\hat{\tilde{\theta}}$ & $\bar{\omega}$ & そ̊ & $\bar{\varnothing}$ & 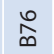 & \&̊ㅜㅇ & N & ঠ゚ & $\underset{\simeq}{\pi}$ \\
\hline
\end{tabular}


- Partitionen in \% (Verteilung über die Partitionen)

Eine MDC kann in drei Partitionen aufgeteilt sein:

- DRGs liegen in der chirurgischen Partition, wenn sie eine Prozedur beinhalten, für die ein OP-Saal erforderlich ist.

- DRGs der anderen Partition beinhalten Prozeduren, die in der Regel diagnostische Maßnahmen abbilden und für die kein OP-Saal erforderlich ist.

- DRGs der medizinischen Partition beinhalten keine relevanten Prozeduren.

Die Abkürzungen der Partitionen bedeuten Folgendes:

o = operativ

$\mathrm{a}=$ andere

$\mathrm{m}=$ medizinisch

\section{- Budget-Anteile ZE/SE}

Für Leistungen, die mit DRGs noch nicht sachgerecht vergütet werden, können die Vertragspartner individuelle Leistungskomplexe und Entgelte vereinbaren. Dazu gehören im Jahr 2017 u. a. 45 DRGs (davon drei teilstationäre), zu denen keine sachgerechte Bewertungsrelation durch das InEK ermittelt werden konnte, aber auch Leistungen in besonderen Einrichtungen und teilstationäre Behandlung. ${ }^{3}$ Die Spalte Budgetanteil SE beschreibt den Anteil solcher tages- oder fallbezogenen Leistungen am Gesamtbudget aus DRGs, Zusatzentgelten und sonstigen Entgelten. Dieser Budgetanteil ist von der Vergütung nach DRGs sowie der Budgetkonvergenz ausgenommen.

Zusatzentgelte können neben DRG-Fallpauschalen sowie tages- und fallbezogenen sonstigen Entgelten zusätzlich abgerechnet werden. Über die 94 vom InEK kalkulierten und bundeseinheitlich vergüteten hinaus können weitere hausindividuelle Zusatzentgelte vereinbart werden.

\section{- Bes. Leist. (B/N/H/P)}

In mit einem „B“ gekennzeichneten Häusern sind Leistungsbereiche vereinbart, die nach der Vereinbarung zur Bestimmung von Besonderen Einrich-

3 Die Regelungen finden sich im Detail in § 6 Abs. 1 des Krankenhausentgeltgesetzes. tungen - VBE 2017 - von der Abrechnung nach DRG-Fallpauschalen und der Budgetkonvergenz ausgenommen sind. „N“ markiert Einrichtungen, in denen 2017 Entgelte für neue Untersuchungsund Behandlungsmethoden nach $\$ 6$ Abs. 2 des Krankenhausentgeltgesetzes (NUB) vereinbart wurden. „H“ kennzeichnet Krankenhäuser, in denen Zusatzentgelte für hochspezialisierte Leistungen nach $\$ 6$ Abs. 2a des Krankenhausentgeltgesetzes vereinbart wurden. „P“ markiert Krankenhäuser mit einer psychiatrischen Fachabteilung.

\section{- Notfall}

In dieser Spalte findet sich ein „N“, sofern für das Krankenhaus im Jahr 2017 ein Abschlag für die Nichtteilnahme an der Notfallversorgung vereinbart wurde.

\section{- AOK-Patientenwege (PKW-km) (Med/oQ)}

Für jede Einrichtung wird auf Basis der AOK-Krankenhausfälle mit Abrechnung nach Krankenhausentgeltgesetz (KHEntgG) die maximale PKW-Strecke in $\mathrm{km}$ für die $50 \%$ (in der Spalte Med für Median) bzw. 75 \% (in der Spalte oQ für oberes Quartil) der AOK-Versicherten mit der kürzesten Fahrtstrecke dargestellt. Als Startpunkt des Patientenwegs gilt der geografische Mittelpunkt des 5-stelligen PLZ-Gebiets des Patientenwohnorts, als Endpunkt die vollständige Adresse des Krankenhauses.

\section{- Vereinbarte regionale DRG-Marktanteile} und -konzentration im Umkreis von 10, 20 und $30 \mathrm{~km}$ (Marktanteil/HHI)

Die Spalten beschreiben die regionale Markt- und Wettbewerbssituation des jeweiligen Krankenhauses für DRG-Leistungen im Luftlinienumkreis von 10, 20 und $30 \mathrm{~km}$ anhand der Kennzahlen Marktanteil und dem Herfindahl-Hirschman-Index (HHI).

Der ausgewiesene regionale Marktanteil eines Krankenhauses basiert auf den dort konkret vereinbarten Leistungen. Eine Einrichtung in einer Region mit hoher Krankenhausdichte kann also auch einen relativ hohen Marktanteil aufweisen, sofern es Leistungen erbringt, die in der Region ansonsten selten bzw. in geringem Umfang vereinbart sind.

Der Herfindahl-Hirschman-Index ist eine Kennzahl zur Konzentrationsmessung in einem Markt bzw. in einer Marktregion und spiegelt so die 
Wettbewerbsintensität wider. Er ist als Summe der quadrierten Markanteile aller Teilnehmer in einer Region definiert und kann die Werte zwischen 0 und 1 annehmen, wobei der Wert 1 als Synonym für eine Monopolstellung keinem Wettbewerb entspricht. Verteilen sich in einer Wettbewerbsregion die Leistungen gleichmäßig auf zwei Anbieter, so haben beide einen Marktanteil von $50 \%$, der quadrierte Marktanteil beträgt jeweils 0,25 und der HHI als Summe der quadrierten Marktanteile ist 0,50 . Verteilen sich die Leistungen aber nicht gleichmäßig auf die zwei Anbieter, sondern im Verhältnis $99 \%$ zu $1 \%$, so nimmt der HHI einen Wert in der Nähe von 1 ein und spiegelt so die monopolistische Angebotsstruktur wider.

Um unerwünschte Effekte aus noch nicht geschlossenen Vereinbarungen zu minimieren, basieren die Marktdaten abweichend von den übrigen Werten in der Tabelle aus der Vorjahres-Budgetrunde.

\section{- Infozeile Bundesland}

Die Darstellung ist sortiert nach Bundesländern und dem Namen des Standortes. Für jedes Bundesland werden in einer Zeile die gewichteten Mittelwerte CMI, Anteile der Partitionen an Gesamtfällen, Leistungsdichte Basis-DRG, Top MDC, Budgetanteile von Zusatzentgelten und sonstigen Entgelten sowie die Anzahl der Krankenhäuser mit vereinbarten besonderen Leistungen dargestellt.

\section{- QSR-Behandlungsergebnisse}

Das QSR-Verfahren der AOK ist ein Verfahren zur Qualitätsmessung von Krankenhausbehandlungen. Die Abkürzung QSR steht für „Qualitätssicherung mit Routinedaten“. Im QSR-Verfahren kann durch die konsequente Analyse der Behandlung und des
Überlebensstatus bis zu einem Jahr nach der Erstoperation auch die langfristige Behandlungsqualität gemessen werden. Zur Berechnung der Qualitätsindikatoren werden Abrechnungs- bzw. Routinedaten verwendet. Diese werden den Krankenkassen automatisch vom Krankenhaus übermittelt, um die Behandlung eines Patienten in Rechnung zu stellen, oder liegen der Krankenkasse bereits in den Versichertenstammdaten vor.

Im Krankenhaus-Directory stehen die krankenhausbezogenen Ergebnisse für folgende Leistungsbereiche zur Verfügung: Einsetzen einer Endoprothese oder osteosynthetische Versorgung nach einem hüftgelenknahen Oberschenkelbruch, Einsetzen einer Hüftendoprothese bei Coxarthrose (Hüft-EP), Einsetzen eines künstlichen Kniegelenks bei Gonarthrose (Knie-EP), Gallenblasenentfernung bei Gallensteinen, Blinddarmentfernung, Operation bei gutartiger Prostatavergrößerung, Prostataentfernung bei Prostatakrebs und therapeutische Herzkatheter (PCI) bei Patienten ohne Herzinfarkt. Das aktuelle Verfahrensjahr 2017 umfasst den Berichtszeitraum 2014 bis 2016 mit 2017 zur Nachbeobachtung der Patienten.

Die klinikbezogenen QSR-Ergebnisse werden auch im AOK-Krankenhausnavigator auf Basis der Weißen Liste frei zugänglich veröffentlicht. ${ }^{4}$

\section{Literatur}

Friedrich J, Leber WD, Wolff J (2010) Basisfallwerte - zur Preisund Produktivitätsentwicklung stationärer Leistungen. In: Klauber J, Geraedts M, Friedrich J (Hrsg) Krankenhaus-Report 2010. Schattauer, Stuttgart, S 122-147

4 www.aok.de/krankenhausnavi

Open Access Dieses Kapitel wird unter der Creative Commons Namensnennung 4.0 International Lizenz(http://creativecommons. org/licenses/by/4.0/deed.de) veröffentlicht, welche die Nutzung, Vervielfältigung, Bearbeitung, Verbreitung und Wiedergabe in jeglichem Medium und Format erlaubt, sofern Sie den/die ursprünglichen Autor(en) und die Quelle ordnungsgemäß nennen, einen Link zur Creative Commons Lizenz beifügen und angeben, ob Änderungen vorgenommen wurden.

Die in diesem Kapitel enthaltenen Bilder und sonstiges Drittmaterial unterliegen ebenfalls der genannten Creative Commons Lizenz, sofern sich aus der Abbildungslegende nichts anderes ergibt. Sofern das betreffende Material nicht unter der genannten Creative Commons Lizenz steht und die betreffende Handlung nicht nach gesetzlichen Vorschriften erlaubt ist, ist für die oben aufgeführten Weiterverwendungen des Materials die Einwilligung des jeweiligen Rechteinhabers einzuholen. 